\title{
Group Title: Functional outcomes among patients with acute ischemic stroke after mechanical thrombectomy with or without intravenous thrombolysis
}

In their randomized clinical trial, Suzuki and colleagues1 reported that mechanical thrombectomy alone failed to demonstrate noninferiority when compared to combined intravenous thrombolysis and mechanical thrombectomy with regard to favorable functional outcome at 90 days. We have some concerns about this study.

First, several baseline characteristics of the patients were not well balanced in the treatment groups, as shown in the Table 1 . For example, $55 \%$ of the patients in the mechanical thrombectomy alone group were men vs $70 \%$ in the combined intravenous thrombolysis and mechanical thrombectomy group. Although imbalances between groups can occur by chance in a randomized clinical trial, it is unusual to have imbalances for several baseline variables simultaneously, particularly for important prognosis factors in patients with stroke, such as blood pressure, stroke severity and stroke location.

Therefore, we are concerned about systematic bias from the randomization process in this open-label trial.

Second, although the discrepancy between the primary outcome selection in the initial and final protocol was explained in the publication, the study hypothesis was not clearly stated in the protocol, and did not follow the SPIRIT guidance. ${ }^{2}$ Additionally, the sample size description in both final protocol and statistical analysis plan indicated that 178 patients were required, assuming a favorable outcome in $48.6 \%$ with mechanical thrombectomy alone and $35.2 \%$ with combined intravenous thrombolysis and mechanical thrombectomy. The non-inferiority margin for the primary outcome was not predefined or considered in the sample size calculation for this "non-inferiority" trial but was compared against an odds ratio of 0.74 , derived from a previous meta-analysis. Also, the 
non-inferiority margin was not clearly prespecified but was tested for the secondary outcome of modified Rankin Scale score reduction.

Finally, the percentage of patients projected to drop out of this study decreased from $33 \%$ in the initial study design to $11 \%$ in the final study design. Due to the above concerns, we believe further clarifications and justifications are needed to avoid misinterpreting the findings of this trial.

Hui Mai, MD, PhD

Department of Neurology

Affiliated Hospital of Guangdong Medical University

Zhanjiang, China

Tao Chen, $\mathrm{PhD}$

Department of Clinical Sciences

Liverpool School of Tropical Medicine

Liverpool, United Kingdom

Bin Zhao, MD

Department of Neurology

Affiliated Hospital of Guangdong Medical University

Zhanjiang, China

Correspondence Author: Bin Zhao, MD, Department of Neurology, Affiliated Hospital of Guangdong Medical University, NO.57 Renmingdadaonan, Xiashan district, Zhanjiang, 524023, Guangdong, China. (binzhaoe@163.com)

Conflict of Interest Disclosures: None reported.

1.Suzuki K, Matsumaru Y, Takeuchi M, et al. Effect of mechanical thrombectomy without vs with intravenous thrombolysis on functional outcome among patients with acute ischemic stroke: the SKIP randomized clinical trial. JAMA. 2021;325(3):244-253. doi: 10.1001/jama.2020.23522

2.Chan AW, Tetzlaff JM, Gotzsche PC, et al. SPIRIT 2013 explanation and elaboration: guidance for protocols of clinical trials. BMJ. 2013;346:e7586. doi: 10.1136/bmj.e7586 